\title{
Direct thermal imaging of circumstellar discs and exo-planets
}

\author{
Eric Pantin ${ }^{a}$, Ralf Siebenmorgen ${ }^{b}$, Celine Cavarroc $^{a}$, Michael F. Sterzik ${ }^{c}$ \\ ${ }^{a}$ Laboratoire AIM, CEA/DSM - CNRS - Universit Paris Diderot, IRFU/SAp, France; \\ ${ }^{b}$ European Southern Observatory, Karl-Schwarzschildstr. 2, 85748 Garching, Germany; \\ ${ }^{c}$ European Southern Observatory, Chile;
}

\begin{abstract}
The phase A study of a mid infrared imager and spectrograph for the European Extremely Large Telescope (E-ELT), called METIS, was endorsed in May 2008. ${ }^{1}$ Two key science drivers of METIS are: a) direct thermal imaging of exo-planets and b) characterization of circumstellar discs from the early proto-planetary to the late debris phase. Observations in the $10 \mu \mathrm{m}$ atmospheric window ( $\mathrm{N}$ band) require a contrast ratio between stellar light and emitted photons from the exo-planet or the disc of $\sim 10^{5}$. At shorter wavelengths the contrast between star and reflected light from the planet-disc system exceeds $\gtrsim 10^{7}$ posing technical challenges. By means of endto-end detailed simulations we demonstrate that the superb spatial resolution of a $42 \mathrm{~m}$ telescope in combination with stellar light rejection methods such as coronagraphic or differential imaging will allow detections at $10 \mu \mathrm{m}$ for a solar type system down to a star-planet separation of $0.1^{\prime \prime}$ and a mass limit for irradiated planets of 1 Jupiter $\left(\mathrm{M}_{\mathrm{J}}\right)$ mass. In case of self-luminous planets observations are possible further out e.g. at the separation limit of JWST of $\sim 0.7^{\prime \prime}$, METIS will detect planets $\gtrsim 5 \mathrm{M}_{\mathrm{J}}$. This allows to derive a census of all such exo-planets by means of thermal imaging in a volume limited sample of up to $6 \mathrm{pc}$. In addition, METIS will provide the possibility to study the chemical composition of atmospheres of exo-planets using spectroscopy at moderate spectral resolution $(\lambda / \Delta \lambda \sim 100)$ for the brightest targets. Based on detailed performance and sensitivity estimates, we demonstrate that a mid-infrared instrument at an ELT is perfectly suited to observe gravitationally created structures such as gaps in proto- and post- planetary discs, in a complementary way to space missions (e.g. JWST, SOFIA) and ALMA which can only probe the cold dust emission further out.
\end{abstract}

Keywords: thermal infrared, mid infrared, exo-planet, proto-planetary discs, debris discs, coronagraphy

\section{INTRODUCTION}

Since the first identification of a planet outside the solar system ${ }^{2}$ more than 200 exo-planets have been discovered, the vast majority by means of precise radial velocity measurements (The Extrasolar Planet Encyclopaedia presents a comprehensive list of all known exoplanets. It is available at the following URL:

http://www.obspm.fr/planets). The direct detection of exo-planets is of wide public interest because philosophical questions concerning possible existence of extra-terrestrial life are linked. In modern astronomy theories related to the formation and origin of planetary systems require input which can only be obtained from direct imaging of such systems. Therefore direct imaging of exo-planets is taken as one key science driver for the instrumentation suite of many next generation large telescopes, including the E-ELT.

Protoplanetary discs are nurseries in which the planet embryos form. Once a sufficiently massive proto-planet exists in the disc and before eventual migration a gap in the disc structure opens. ${ }^{3}$ Although based on slightly different physics, the gravitational interaction of the planet with dust particles produces a footprint in the density structure of the debris disc. While direct imaging of exo-planets is limited by the relatively modest sensitivity of ground-based observations a study of the disc structure offers another although indirect detection method of exo-planets. It has the advantage to be applicable to lower mass planets located further out in the disc or to planetary systems at larger distances from Earth.

For direct and indirect exo-planet detection strategies a mid-infrared instrument mounted at an ELT like METIS offers a unique combination of a relatively low contrast between star and the emission from the planetary disc system at ultimate sharp angular resolution.

In the following sections a description of our modeling efforts is first given. Concerning exo-planets direct observations, simulated data and performances are shown; sensitivity limits in terms of minimum mass as a function 
of the distance to the star are assessed, either using direct imaging, or using four quadrants phase mask (4 QPM) coronagraphs. In the case of proto-planetary discs and debris discs, relevant simulations are shown to prove that planet-induced footprints are easily detectable when using 4 QPMs coronagraphs as well.

\section{PSF MODEL}

Imaging the dust density structure of circumstellar discs or the direct detection of exo-planets requires precise understanding of the point spread function (PSF) of the observing system. In the modeling efforts we consider PSF computed using present knowledge of the E-ELT pupil design. Assumed properties are a segmented M1 with $42 \mathrm{~m}$ and an adaptive optics systems with cut-off frequency at $500 \mathrm{~Hz}$ and $84 \times 84$ actuators of the deformable mirror. In the simulations a Monte Carlo scheme is applied in which an ad-hoc degradation of the AO corrected power-spectrum of the wavefront error is included to simulate e.g. the static aberrations also as any other source of AO performances degradation. This provides a set of short exposure phase maps which after averaging gives a reasonable representation of the long-exposure PSF $(\sim 1 \mathrm{~h})$ which have Strehl ratii in the range 0.93-0.95 at $10 \mu \mathrm{m}$.

In Fig. 2 and Fig. 3 we show the residual noise, $\sigma_{\mathrm{R}}$, derived for different observing techniques as function of distance from the star. The residual noise is estimated from azimuthal averages of the image. One notice that $\sigma_{\mathrm{R}}$ always exceeds the background noise, $\sigma_{\mathrm{B}}$, up to large angular separations $\left(\gtrsim 2^{\prime \prime}\right)$ from the star. For the instrument we assume background noise limited performances. In case of a direct image without further contrast enhancement the noise from the stellar profile exceeds the background noise by $\sim 5$ orders of magnitude at $0.1 "$.

Computing PSF under different seeing conditions allows to estimate residuals after star subtraction. We assumed seeing variations of $0.6^{\prime \prime}$ and $0.7^{\prime \prime}$ between two $1 \mathrm{~h}$ long observations, respectively. Standard subtraction techniques using a reference star image provide only some advantages compared to direct imaging at separations $\lesssim 0.1^{\prime \prime}$ but not further out. This is because seeing induced PSF variations are mainly located in the wings of the PSF. Again, the residuals noise exceeds the background noise by a very large factor (see e.g. Fig.2).

Significant contrast enhancement can be achieved by using a coronagraph. In the simulations we applied a four quadrants phase mask (4QPM) as baseline for which the 4QPM model (rouan2000) is in good agreement with existing performance tests of the real system. As above for standard PSF subtraction techniques we utilize reference star observations with such a coronagraph at seeing variation of $0.6^{\prime \prime}$ and $0.7^{\prime \prime}$ and subtract both coronagraphic images from each other. The use of a coronagraph reduces the residual noise by 1 or even 2 orders of magnitude as compared to standard techniques (Fig. 4 and Fig.5). In addition the use of a coronagraph prevents the appearance of other spurious artifacts caused by detector saturation. Note that the 4QPM simulations have been carried out at a single wavelength, assuming a $\mathrm{R}=10$ imaging filter. Other types of achromatic coronagraphs have to be studied in combination with e.g. differential imaging, to push further the sensitivity limits.

\section{DIRECT THERMAL IMAGING OF EXO-PLANETS}

The direct imaging of a exo-planet remains challenging with present day technology. This can be understood by inspecting the solar system. In Fig.1, the contrast in peak brightness between the Sun and Jupiter is $5 \times 10^{10}$ in the optical (V band), some $10^{9}$ in the NIR (J band) and diminishes to a few $10^{6}$ in the thermal infrared ( $\mathrm{N}$ band). Another important advantage of the thermal infrared in particular around $10 \mu \mathrm{m}$ is that such long waveband observations are accompanied by much better behaved PSF with high Strehl ratios and reduced requirements on the performance of the AO system. The expected Strehl ratios provided by an E-ELT AO system are of the order of $\sim 65 \%$ in $\mathrm{H}$ band but exceeding $>90 \%$ in the $\mathrm{N}$ band. Furthermore, the thermal infrared is quite complementary to shorter wavelength observations. While in the latter exo-planets are detected from reflected star light one observes in the mid infrared the direct thermal emission of the stellar heated or self-luminous planet. The distinct detection mechanisms in the near and mid infrared are of utmost importance for characterizing the physical and chemical properties of the exo-planets and their atmospheres. 


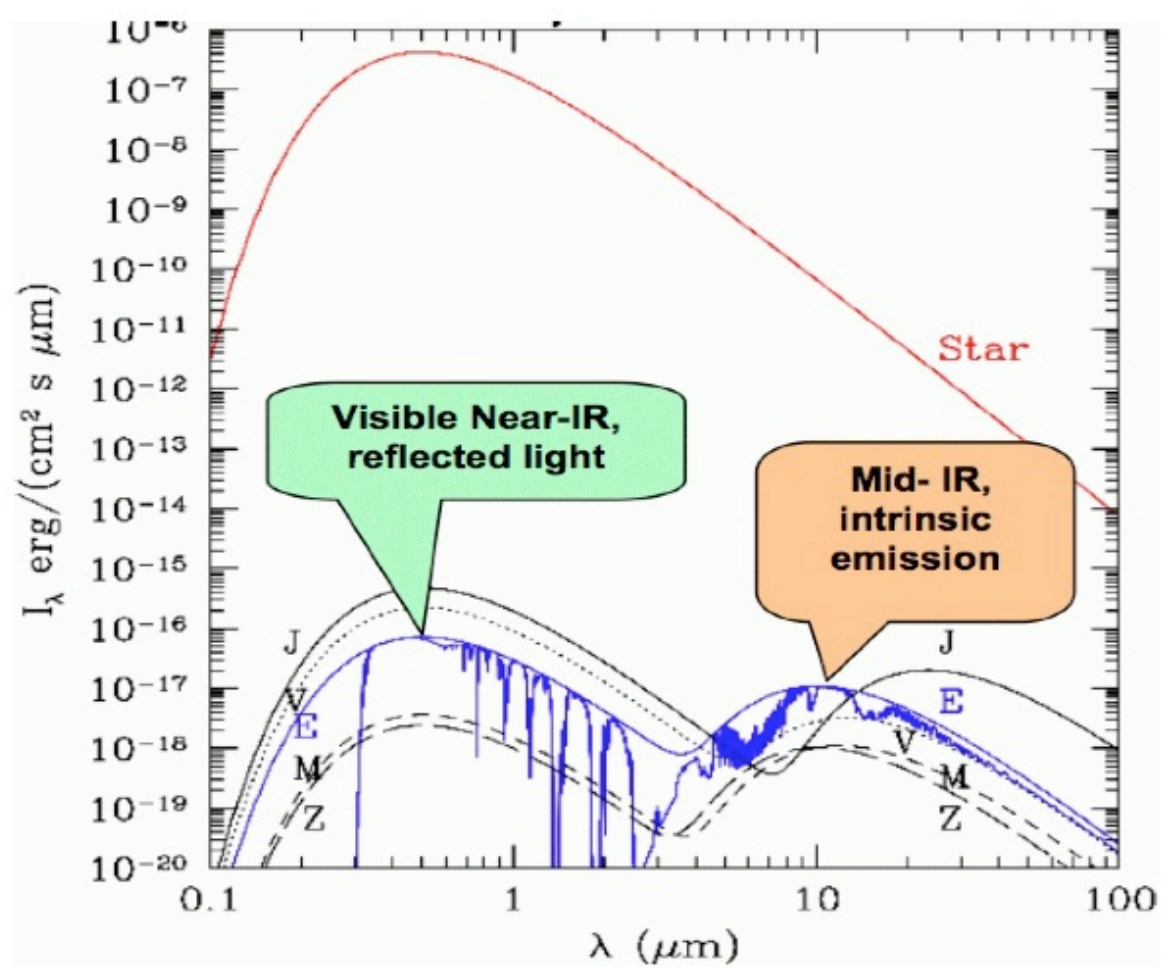

Figure 1. Comparison between the flux emitted by the Sun (a G2V star shown in red) and those (black lines) coming from the planets of the solar system assumed to be black-body emitters(J=Jupiter, V=Venus, E=Earth, M=Mars). Z represents the spectral distribution of the zodiacal light. The blue line is a more realistic emission model of Earth.

\subsection{Detection likelihood for super-Earths}

The so-called super-Earths are probably the most massive telluric planets that nature can form, ${ }^{4}$ some of them being discovered within the habitable zone. ${ }^{5}$ Except for some exceptional physical circumstances a rocky planetary core of mass 10 times the Earth will attract the surrounding material of the disc and finally form a giant gaseous planet. ${ }^{6}$ Super-Earth like planets are therefore limited to a maximum of 10 Earth masses which translates to a upper limit of the radius for super-Earths of less than twice the Earth radius.

For estimating the emission of a super-Earth we consider an irradiated planet*. To first order the planet flux can be derived from a black-body heated by a star of luminosity $L$ and located at an orbital separation $r$ to a temperature: $T^{4} \propto L / r^{2}\left({ }^{7}\right)$. Therefore irradiated exo-planets such as super-Earths become brighter with increasing stellar luminosity and smaller separation from the star. However, increasing star brightness results in an increase of the residual noise level from stellar light rejection (Sect. 2) so that the planet detection becomes unfortunately also more challenging. For main-sequence stars and including a 4QPM coronagraph in the simulations we find that in any configuration of stellar type and orbital separation a super-Earth like exoplanet is most likely below the detection threshold at an E-ELT. This prediction is either caused by the expected sensitivity of a background noise limited instrument which is for the E-ELT of order $\sim 20 \mu \mathrm{Jy} / 10 \sigma / 1 \mathrm{~h}$ in the N band for a site similar to Paranal or by the achievable contrast at small separation form the star which is given by the residual noise floor of the stellar light rejection.

\subsection{Giant gaseous planets}

Two types of models are considered to estimate detection thresholds of giant gaseous planets. In the first an irradiated giant planet is supposed to be in close orbit of $0.2-1 \mathrm{AU}$ from the star and in the second we take self-

\footnotetext{
${ }^{*}$ Irradiated planets are exclusively heated by the star and have no self-luminosity.
} 
luminous giant planets at distances further out: 3-10AU. Planetary model atmospheres are taken for irradiated planets from Barman et al. ${ }^{8}$ and self-luminous planets from Burrows et al. ${ }^{9}$

The star is $\sim 10^{5}$ times brighter than giant exo-planets and stellar light rejection as discussed in Sect.2 is an essential issue. Fig.2 and Fig.3 show : i) the azimuthally averaged profile the star, ii) the detection limits (100 times the standard deviation of the residuals) after subtraction by a reference star and iii) detection limits after subtraction of two coronagraphic stellar images. PSFs have been computed for observing time of $1 \mathrm{~h}$ and seeing of $0.6^{\prime \prime}$ and $0.7^{\prime \prime}$. The detection threshold $(10 \sigma)$ set by the background noise limit for a $1 \mathrm{~h}$ integration at Paranal is also indicated.

Around solar-type stars $(\mathrm{G}-\mathrm{K})$ with luminosity $L=1 \mathrm{~L}_{\odot}$, temperature $T=5780 \mathrm{~K}$ irradiated giant planets of 1 Jupiter mass are detected in coronagraphic images as close as $\sim 0.1^{\prime \prime}$ from the star. We considered in the models a 5pc distance from Earth. The expected flux of $\sim 25 \mathrm{mJy}$ of an irradiated giant planet at a distance of 0.5 AU from a G-type star is remarkably strong. Therefore, detection of such planets is in principle possible up to fairly large distances of $\sim 50 \mathrm{pc}$ from Earth when extrapolating the quantities of Fig.2. Further away the flux of the planet starves in the background noise. The reasons to explain these remarkable detection performances are twofold. First, the radius of an irradiated giant planet is weakly dependent on the mass or the age. ${ }^{10}$ Second, the temperature of an irradiated exo-planet, mainly determined by the stellar flux and the orbital distance, is considerably higher than the temperature of self-luminous planets.

For self-luminous exo-planets, depending in the site and the observing time, the minimum masses are, assuming a distance of 5 pc from Earth :

- For a $\mathrm{G} 2 \operatorname{star}\left(\mathrm{T}_{e f f}=5780 \mathrm{~K}, \mathrm{~L}=1 \mathrm{~L}_{\odot}\right)$ :

$-7 \mathrm{M}_{j}$ for $\mathrm{a} \geq 1 \operatorname{arcsec}$

- $2 \mathrm{M}_{j}$ marginally detectable at 2 arcsec and more

where $\mathrm{a}$ is the orbital distance of the planet.

- For a M5 star $\left(\mathrm{T}_{e f f}=2800 \mathrm{~K}, \mathrm{~L}=0.01 \mathrm{~L}_{\odot}\right.$, see Fig.3):

$-2 \mathrm{M}_{j}$ at $1.0 \operatorname{arcsec}$

$-5 \mathrm{M}_{j}$ at 0.1 arcsec

Fig.4,5,6 display examples of simulated imaging observations of 1 Gyr old, 1-10 Jupiter mass giant exo-planets with orbital distances in the range 1-4 AU around a solar-type G2V and a M5 later type stars. The observations are carried out in N-band at $8.6 \mu \mathrm{m}$ where one gets an optimum combination of planet flux/sensitivity/spatial resolution. The simulations include the effects of sky shot noise and PSF variations with the seeing.

More advanced PSF suppression, using i.e. differential techniques like spectral differential imaging (SDI) which allow to reduce considerably PSF variations are foreseen strategies to even improve the detection thresholds.

\subsection{Low-resolution spectroscopy}

In Fig.7 we summarize up to which limiting distance one can expect to perform MIR low-resolution spectroscopy depending on the companion mass, assuming a sensitivity limit of $1 \mathrm{mJy} / 10 \sigma / 1 \mathrm{~h}$. In this case we assume a solar analogue as illuminating source. Clearly, rather young host stars are preferred. Intense efforts to find and characterize young stars near the sun have already been pursued (see Ref.11), and suggest promising targets, e.g. about 50 stars located in the $\beta$ Pictoris association, estimated to be only 10-15 Myrs old at a mean distance of $31 \mathrm{pc}$. 


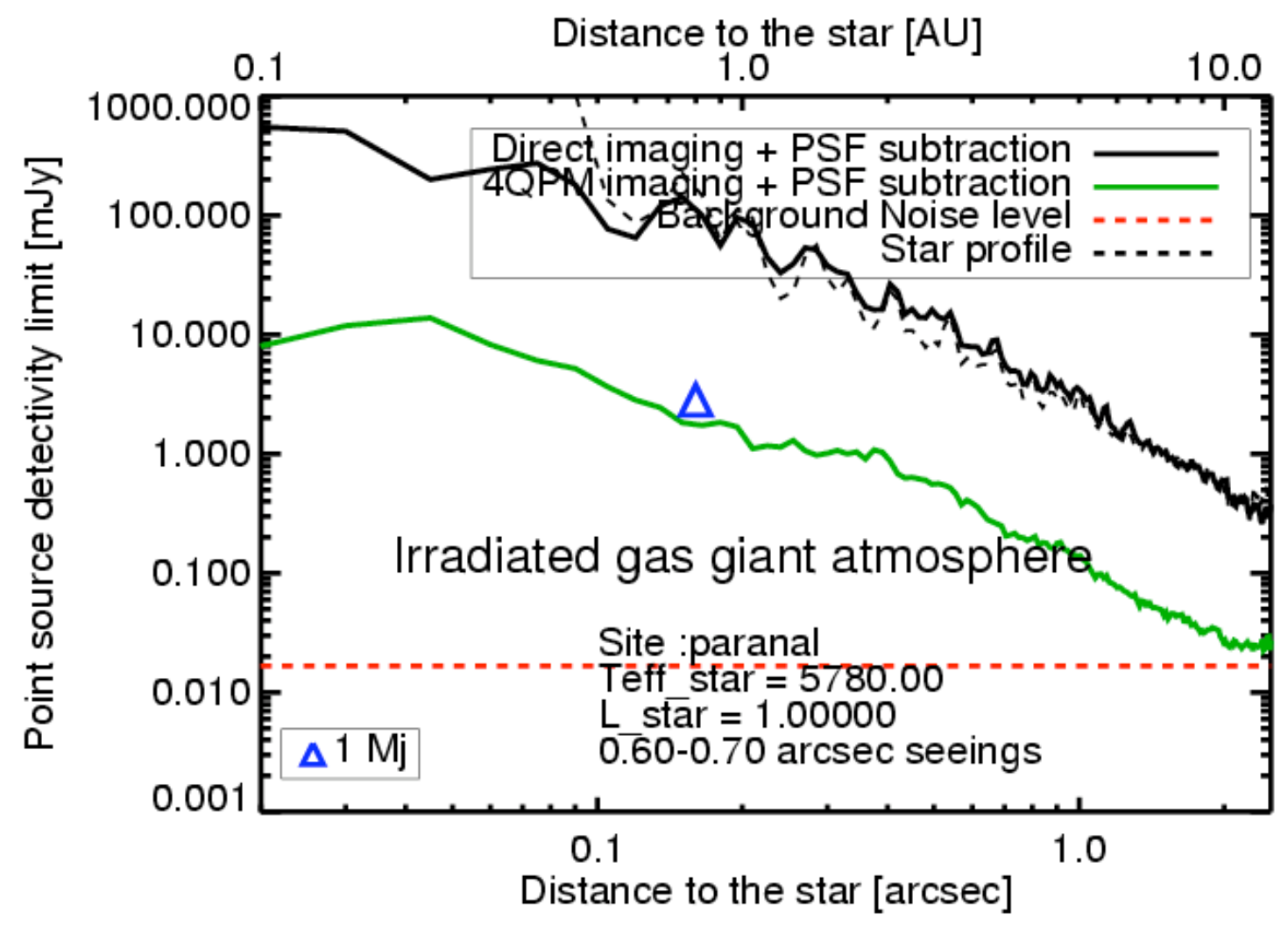

Figure 2. Detection limit (set experimentally to 100 times the residuals standard deviation) as a function of angular distance from $\mathrm{G} 2 \mathrm{~V} \operatorname{star}(L=1 \mathrm{~L} \odot, T=5780 \mathrm{~K})$ is shown for: i) the direct stellar profile (black dashed), ii) as derived by standard star subtraction (black) and iii) after subtraction of coronagraphic stellar images utilizing a 4QPM (green). PSFs have been computed for observing time of $1 \mathrm{~h}$ and seeing of $0.6^{\prime \prime}$ and $0.7^{\prime \prime}$, respectively. The detection threshold $(10 \sigma)$ set by the background noise limit for a $1 \mathrm{~h}$ integration at Paranal is indicated (red dashes). The expected flux of a 1 Jupiter mass irradiated exo-planet is marked by the blue triangle. 


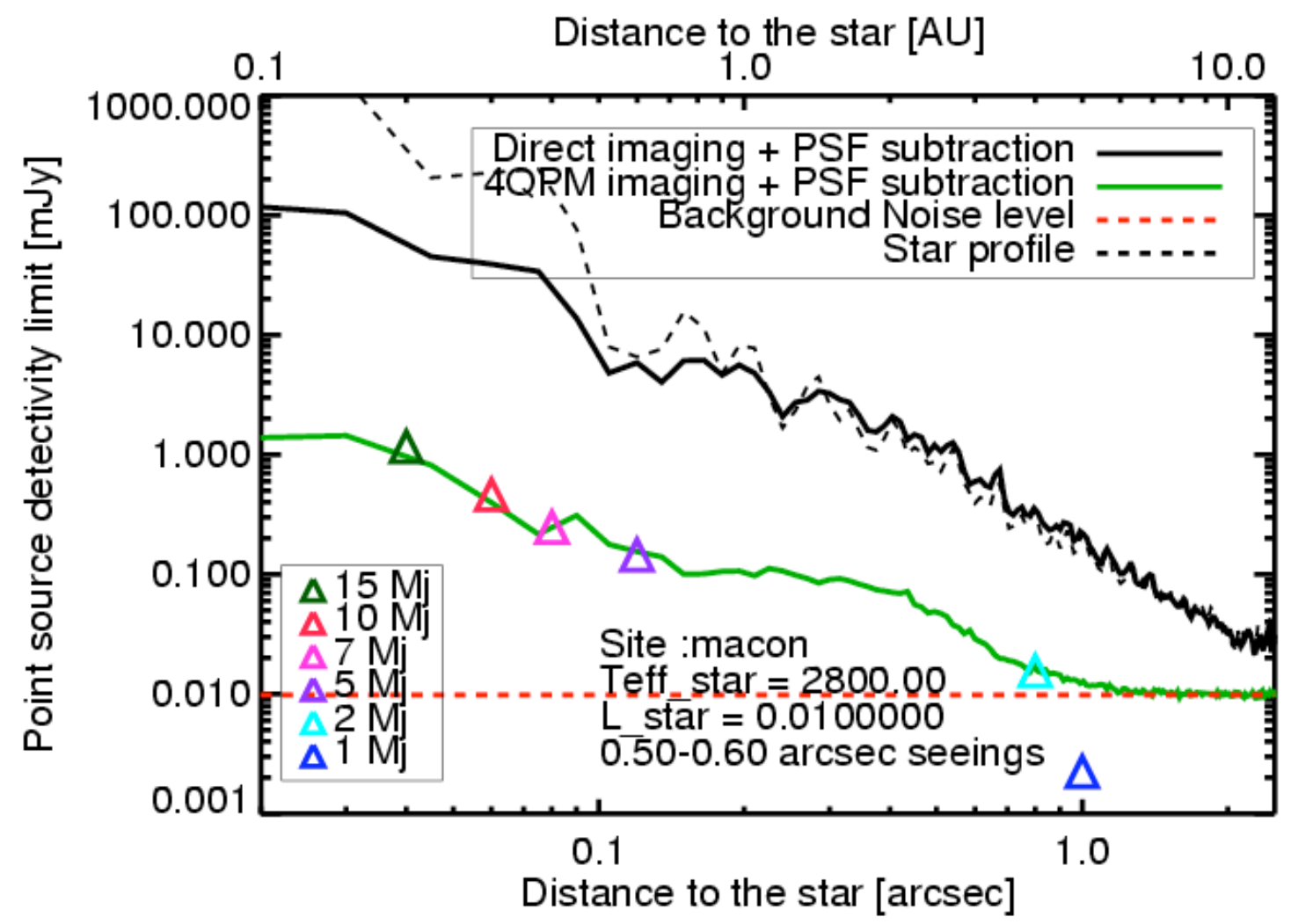

Figure 3. Detection limit (set experimentally to 100 times the residuals standard deviation) as a function of angular distance from M5 star $(L=0.01 \mathrm{~L} \odot, T=2800 \mathrm{~K})$ is shown for: i) the direct stellar profile (black dashed), ii) as derived by standard star subtraction (black) and iii) after subtraction of coronagraphic stellar images utilizing a 4QPM (green). PSF have been computed for observing time of $3 \mathrm{~h}$ and seeing of $0.5^{\prime \prime}$ and $0.6^{\prime \prime}$, respectively. The detection threshold $(10 \sigma)$ set by the background noise limit for a $3 \mathrm{~h}$ integration at Macon is indicated (red dashes). The expected flux of self-luminous giant exo-planets are marked for masses between 1 and 15 times of Jupiter (triangular). 


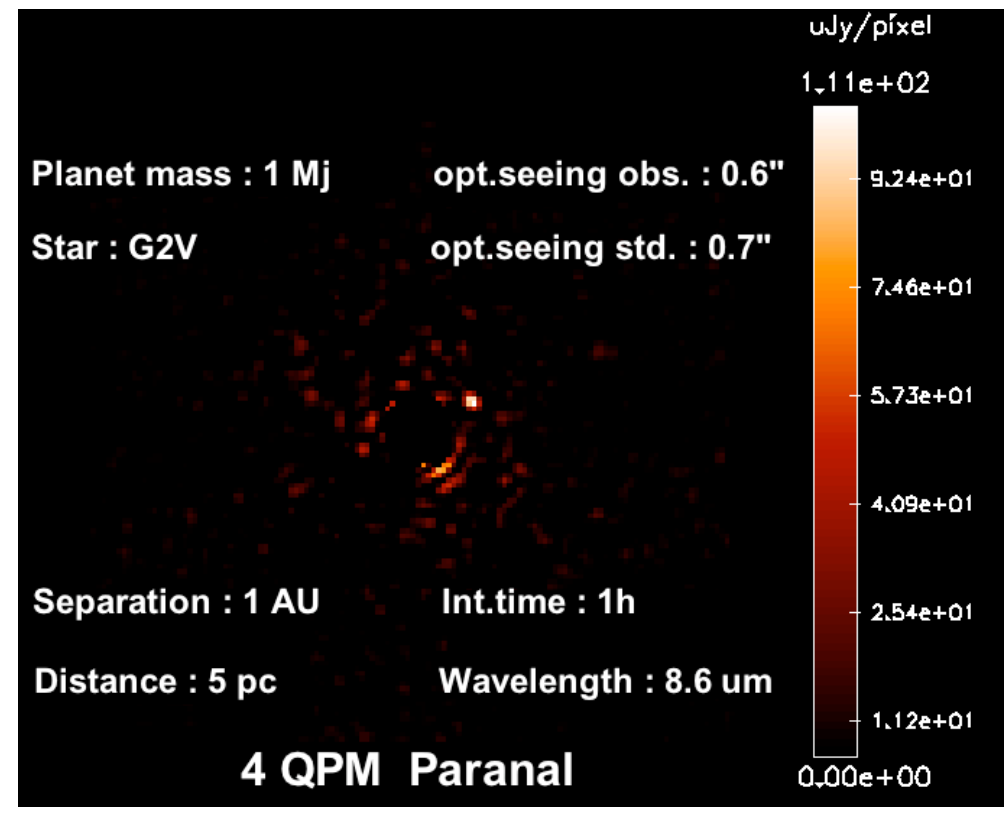

Figure 4. Simulated image observations of a 1 Gyr old, $1.234 \mathrm{R}_{j}$ and $1 \mathrm{M}_{j}$ irradiated planet at $1 \mathrm{AU} / 5 \mathrm{pc}$ around a G2V star. The parameters assumed for the simulated are displayed on the images. Inner $(\mathrm{r} \leq 0.1$ ") regions have been masked.

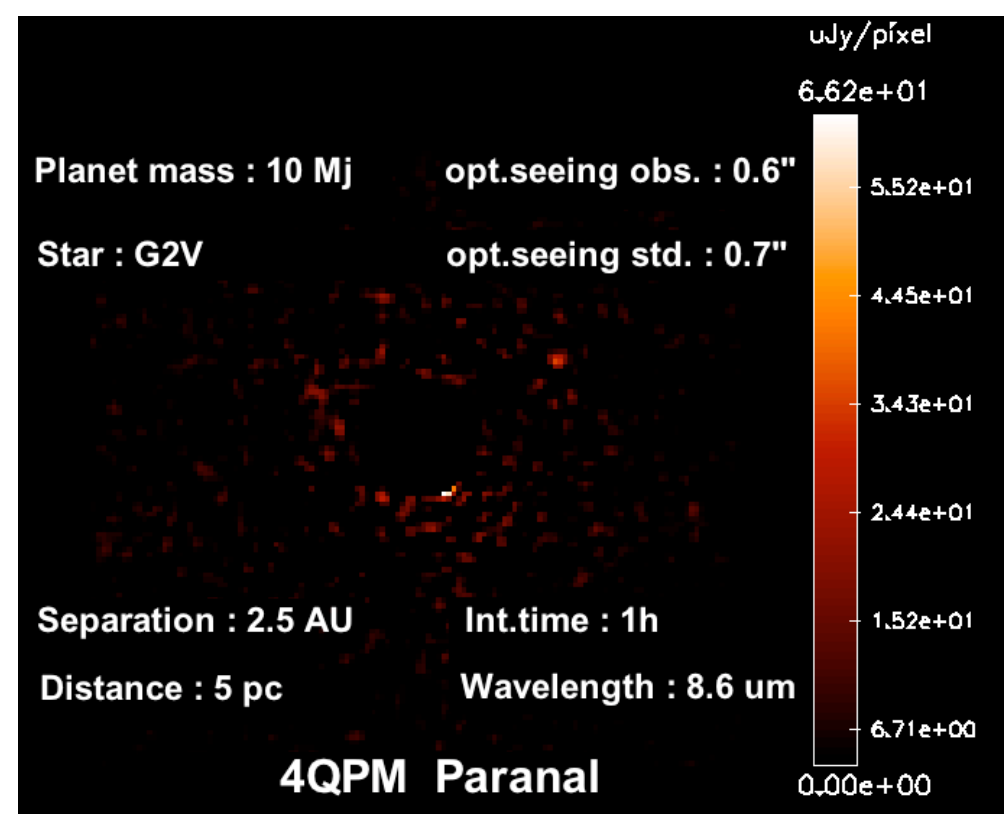

Figure 5. Simulated image observations of a $1 \mathrm{Gyr}$ old, $10 \mathrm{M}_{j}$ planet at $2.5 \mathrm{AU} / 5 \mathrm{pc}$ around a G2V star. Inner (r $\leq 0.2$ ") regions have been masked. 


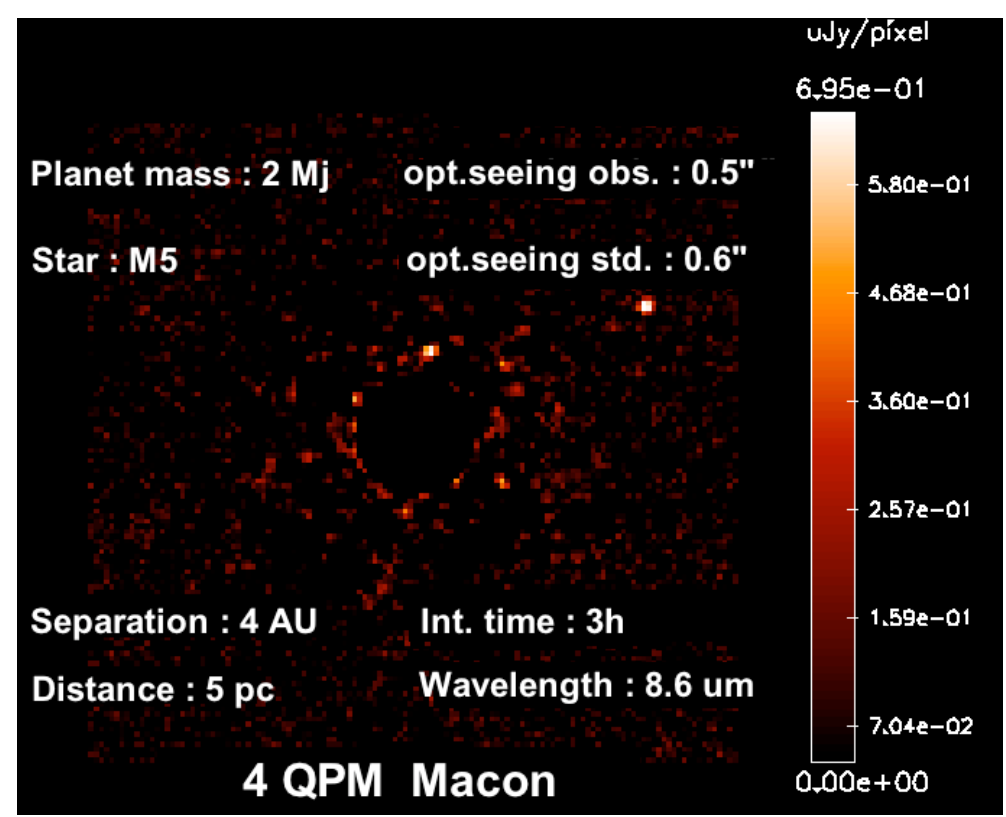

Figure 6. Simulated image observations of a 1 Gyr old, $2 \mathrm{M}_{j}$ planet at $4.0 \mathrm{AU} / 5 \mathrm{pc}$ around a M5 star. Inner (r $\left.\leq 0.2 "\right)$ have been masked.

\section{DUSTY DISCS AND EXO-PLANETS}

Based on the large number of known planetary systems and on the wealth of observations of dusty discs around solar-mass young stellar objects, ${ }^{12}$ it is now well established that planets around main-sequence solar-type stars form in massive, gaseous and dusty proto-planetary discs that survive for several million years around the nascent stars. ${ }^{13}$ Dusty discs are thus also targets of highest interest to understand in which physical conditions, with which timescales, exoplanetary systems form from the T-Tauri disc material.

However, very few young stars exist in the close vicinity of the Sun. Closest star forming regions younger than 10 Myr are 50 pc ?? away; the closest T-Tauri star; 51 Oph; is at ?? pc from Earth. The situation is less clear for stars of more than $\sim 2$ solar masses. Such stars have a much higher luminosity than solar-type stars and, according to models, processus like photoevaporation may be at work clearing the inner disc in a few million years. ${ }^{14}$ Whereas radial velocity surveys have just started to reveal planets around stars about twice as massive as the sun, ${ }^{15}$ current imaging observations of proto-planetary discs around stars with such a mass remain very sparse. The lack of well-resolved images of proto-planetary discs around much younger A stars, still on the pre-main-sequence, is due to the fact that massive stars are less numerous than solar-type stars, and in general located farther away than solar-type ones. It will be therefore extremely challenging to perform direct exoplanets detections in young systems at ages of 1-10 millions years at stages at which the giant planets are forming.

However, indirect detections, based on the gravitational interactions between an exoplanet and its surroundings disc material will be achievable given the awaited performances of future instruments. Disc gravitational structuring has been already found in older, main-sequence star, gas-depleted dusty $\operatorname{discs}{ }^{16} / 17 /{ }^{18}$. This structuring has been interpreted in terms of massive planets-induced footprints. Similar gravitational processes are expected to produce gaps and spiral structures in the optically thick, gas supported, proto-planetary discs, ${ }^{3}$ see Fig. 8.

\subsection{Simulations of discs observations using METIS on the E-ELT}

Adopting the same telescope and instrumental models described in Sec.2, we have simulated METIS observations of two types of discs. One is a protoplanetary disc having the same physical parameters as the HD97048 disc; ${ }^{19}$ the second one is debris disc having the same structure as the Beta Pictoris one ${ }^{20}$ but 100 times less massive and placed at $10 \mathrm{pc}$. 


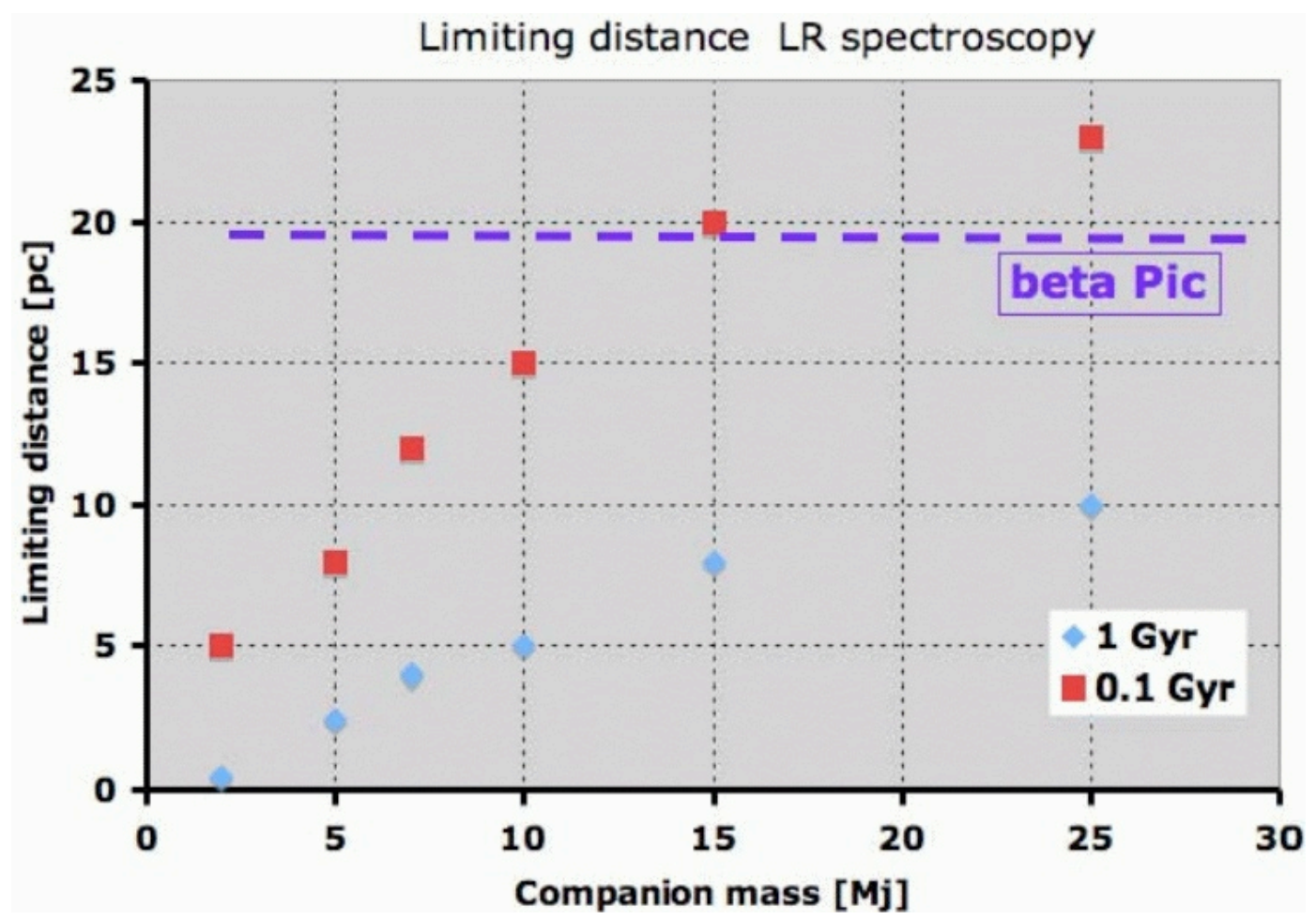

Figure 7. Distance limit, measured from Earth in pc, up to which a exo-planet of given mass, in units of Jupiter mass $M_{\mathrm{J}}$, can be detected in MIR low resolution $(R=\lambda / \Delta \lambda \sim 200)$ mode of METIS. The assumed limiting flux is $1 \mathrm{mJy}$ at Paranal site, for $1 \mathrm{~h}$ exposition and a signal-to-noise ratio of 10 . The emissions of the exo-planet is computed by assuming two ages: 0.1 Gyr (squares) and 1 Gyr (diamonds).

In this simulation we have taken a disc model of emission. in the mid-infrared range. Following Chianf\&Goldreich model, ${ }^{21}$ the surface of the disc is flared. Once the star,the disc geometry and composition of the dust are set, the model uses a full 1D+1D radiative transfer model to compute the output profile of disc emission at a given wavelength (here, $8.6 \mu \mathrm{m}$ ). The model parameters are those of the HD97048 flaring disc given in Ref.19 i.e. : $\mathrm{D}$ (distance to Earth) $=175 \mathrm{pc}, \mathrm{H}$ (scale height at $135 \mathrm{AU})=43 \mathrm{AU}$, Star : $40 \mathrm{~L}_{\odot}$ and 2.5 $\mathrm{M}_{\odot}$. Inner and outer boundary radii of 0.3 au $500 \mathrm{AU}$ respectively. The disc mass is set to $0.01 \mathrm{M}_{\odot}$. The flaring index (of the disc height) is 1.26. For simplicity, the disc is assumed to be observed pole-on.

A simulated gap of matter procued by a giant planet in the disc is added. Its characteristics (depth, width) are computed by the hydro-dynamics code "FARGO" written by F.Masset. ${ }^{22}$ The planet is actually migrating in the disc, but we will assume in the following that the planet has a fixed orbit radius which is true on an $1 \mathrm{~h}$ timescale of observation. Depth and width are then transformed into a local modification of the emission profile using simple physical rules of gas physics local energy balance. Fig.8, right panels, shows the observed profiles of a proto-planetary disc emission derived from the model described above. A 1 Jupiter mass exoplanet has been put in the disc at a orbital distance of $20 \mathrm{AU}(0.12 \mathrm{arcsec})$ from the star. The "wiggles" observed on the profiles are due to the Airy pattern in the telescope and instrument PSF. In the first case (black line), direct imaging of object reduces the true gap contrast (initially of a factor $\approx 100$ ) by a factor of $\approx 50$ because of a combination of gap "contamination" by the wings of the central object (either the star or the inner rim emission) and structure smearing by the convolution with the PSF. Note also that the apparition of a gap should in principle also, in a flared disc, lead to the apparition of a "wall"-like heated structure just beyond the gap. A local flux enhancement at $10 \mu \mathrm{m}$ would be thus expected just after the gap. The expected constrast of this structure is of the order of $1 / \cos (\alpha)$ where $\alpha$ is the grazing angle of the star radiation onto the disc flared surface (in addition the local higher warming should in principle in turn, produce locally an increase of the disc height 
i.e. a local "puffing-up"). This flux amplification is not yet implemented in the models shown here. When using a 4 quadrants coronagraph (4 QPM) mask (blue line), the contrast is regained by a typical factor of $\approx 2$.

Simulated observations of a faint debris disc lead to similar conclusions : the use of a 4QPM coronagraphic mask is a valuable help to enhance the contrast of the gravitational structures (in particular, the strongest structure which is the "horseshoe" gap along the planet orbit), see Fig.8.
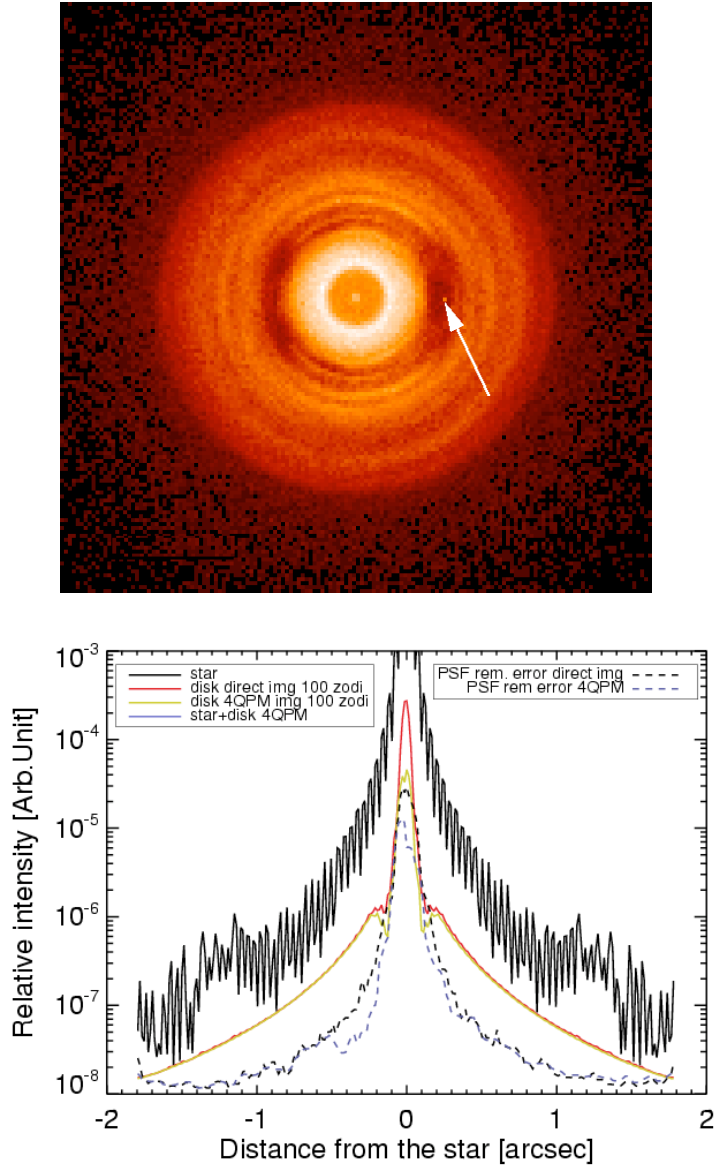
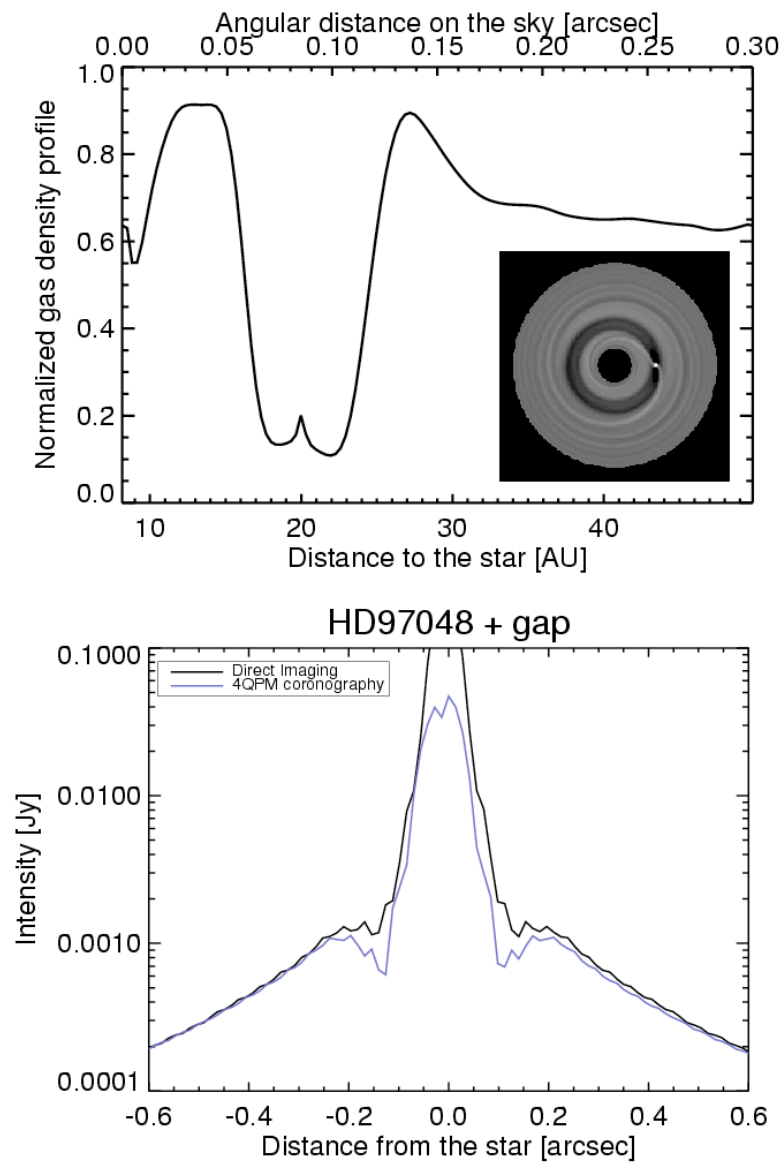

Figure 8. Upper left panel represents the results an example of dynamical simulation of a disc of particles (dust) experiencing radiation pressure from the star (parameter $\beta=0.2$ ); a 1 Jupiter mass planet is embedded (arrow). The planet structures the disc, creating minima and maxima of density. The lower panel shows an observed (using ELT/METIS) debris disc profile. The disc has the same density structure as the $\beta$ Pictoris dust disc, but contains 100 times less mass and is 10 pc away from Earth. A $1 \mathrm{M}_{j}$ planet has been placed on a $2 \mathrm{AU}$ orbit. The use of a four quadrants phase mask coronagraph allows to strengthen contrast of the main "horseshoe" structure observed. The right panels show the same type of simulations, but with a proto-planetary disc similar to HD97048. Upper panel shows the modulation (gap) of the disc density due to a 1 Jupiter mass embedded planet; inset is the disc structure seen pole-on. Lower panel shows the observed disc profiles using the ELT-METIS instrument. The observed gap constrast is also enhanced by $30 \%$ when using a 4QPM coronagraph.

\section{CONCLUSIONS}

We have addressed in this paper the potential of METIS mounted on the E-ELT to make direct imaging of extrasolar giant planets around nearby stars. We show that, depending on the planet type i.e. close-in irradiated or self-luminous one, METIS will be able to directly image a 1 Jupiter mass (respectively a 2 Jupiter masses) on a 1 AU (respectively a 10 AU orbit) for nearby solar-type stars. Around fainter, later-type M5 stars, the minimum 
separation drops to $5 \mathrm{AU}$ for a self-luminous $2 \mathrm{M}_{j}$ planet. METIS is thus a powerful instrument to discover and characterize giant exoplanets are short angular separations $(\alpha \leq 0.5$ ") complementary to MIRI on-board the JWST which is typically limited to $\sim 7 \mathrm{M}_{j}$ at $5 \mathrm{AU}$ (1") for a system $5 \mathrm{pc}$ away.

The E-ELT/METIS instrument is also the perfect tool to study proto- and post- planetary discs. If massive planets are present in a proto-planetary disc, they produce gravitational structures easily detected which will provide us with valuable constraints on planetary formation in the closest star forming regions. In the same order of idea, high angular resolution imaging of closer $(\mathrm{d} \leq 40 \mathrm{pc})$ debris discs is an interesting indirect method of planet detection complementary to the radial velocities one.

The simulations shown in the present paper assume a monochromatic 4QPM coronagraph. The use of future achromatized coronagraphs such as Annular Groove Phase Masks ${ }^{23}$ will allow to combine coronagraphy with differential imaging. In this case, the temporal variations we assumed here are not relevant any longer and the expected performances will be largely improved.

\section{REFERENCES}

[1] Brandl, B., Lenzen, R., Glasse, A., Blommaert, J., Lagage, P., Siebenmorgen, R., and al.., "MIDIR : a mid-infrared instrument for the E-ELT," in [SPIE , Proc. SPIE 7014, id. \#7014M (2008)], Presented at the Society of Photo-Optical Instrumentation Engineers (SPIE) Conference $\mathbf{7 0 1 4}$ (June 2008).

[2] Mayor, M. and Queloz, D., "A Jupiter-Mass Companion to a Solar-Type Star," Nature 378, 355-+ (Nov. 1995).

[3] Artymowicz, P., "Growth and interactions of planets (Invited Review)," in [Planetary Systems in the Universe], Penny, A., ed., IAU Symposium 202, 149-+ (2004).

[4] Rivera, E. J., Lissauer, J. J., Butler, R. P., Marcy, G. W., Vogt, S. S., Fischer, D. A., Brown, T. M., Laughlin, G., and Henry, G. W., "A 7.5 Earth masses Planet Orbiting the Nearby Star, GJ 876," ApJ 634, 625-640 (Nov. 2005).

[5] Udry, S., Bonfils, X., Delfosse, X., Forveille, T., Mayor, M., Perrier, C., Bouchy, F., Lovis, C., Pepe, F., Queloz, D., and Bertaux, J.-L., "The HARPS search for southern extra-solar planets. XI. Super-Earths (5 and 8 earth masses) in a 3-planet system," A\&A 469, L43-L47 (July 2007).

[6] Lissauer, J. J., "Planet formation," ARA\&A 31, 129-174 (1993).

[7] Kruegel, E., [The physics of interstellar dust], The physics of interstellar dust, by Endrik Kruegel. IoP Series in astronomy and astrophysics, ISBN 0750308613. Bristol, UK: The Institute of Physics, 2003. (2003).

[8] Barman, T. S., Hauschildt, P. H., and Allard, F., "Irradiated Planets," ApJ 556, 885-895 (Aug. 2001).

[9] Burrows, A., Sudarsky, D., and Lunine, J. I., "Beyond the T Dwarfs: Theoretical Spectra, Colors, and Detectability of the Coolest Brown Dwarfs," ApJ 596, 587-596 (Oct. 2003).

[10] Fortney, J. J., Marley, M. S., and Barnes, J. W., "Planetary Radii across Five Orders of Magnitude in Mass and Stellar Insolation: Application to Transits," ApJ 659, 1661-1672 (Apr. 2007).

[11] Torres, C. A. O., Quast, G. R., da Silva, L., de La Reza, R., Melo, C. H. F., and Sterzik, M., "Search for associations containing young stars (SACY). I. Sample and searching method," A\&A 460, 695-708 (Dec. 2006).

[12] O'Dell, C. R. and Beckwith, S. V. W., "Young stars and their surroundings.," Science 276, 1355-1359 (1997).

[13] Greaves, J. S., "Disks Around Stars and the Growth of Planetary Systems," Science 307, 68-71 (Jan. 2005).

[14] Takeuchi, T., Clarke, C. J., and Lin, D. N. C., "The Differential Lifetimes of Protostellar Gas and Dust Disks," ApJ 627, 286-292 (July 2005).

[15] Setiawan, J., Rodmann, J., da Silva, L., Hatzes, A. P., Pasquini, L., von der Lühe, O., de Medeiros, J. R., Döllinger, M. P., and Girardi, L., "A substellar companion around the intermediate-mass giant star HD 11977," A\&A 437, L31-L34 (July 2005).

[16] Lagage, P. O. and Pantin, E., "Dust Depletion in the Inner Disk of Beta-Pictoris as a Possible Indicator of Planets," Nature 369, 628-+ (June 1994).

[17] Mouillet, D., Larwood, J. D., Papaloizou, J. C. B., and Lagrange, A. M., "A planet on an inclined orbit as an explanation of the warp in the Beta Pictoris disc," MNRAS 292, 896-+ (Dec. 1997). 
[18] Holland, W. S., Greaves, J. S., Zuckerman, B., Webb, R. A., McCarthy, C., Coulson, I. M., Walther, D. M., Dent, W. R. F., Gear, W. K., and Robson, I., "Submillimetre images of dusty debris around nearby stars," Nature 392, 788-791 (Apr. 1998).

[19] Lagage, P.-O., Doucet, C., Pantin, E., Habart, E., Duchêne, G., Ménard, F., Pinte, C., Charnoz, S., and Pel, J.-W., "Anatomy of a Flaring Proto-Planetary Disk Around a Young Intermediate-Mass Star," Science 314, 621-623 (Oct. 2006).

[20] Augereau, J. C., Nelson, R. P., Lagrange, A. M., Papaloizou, J. C. B., and Mouillet, D., "Dynamical modeling of large scale asymmetries in the beta Pictoris dust disk," A\&A 370, 447-455 (May 2001).

[21] Chiang, E. I. and Goldreich, P., "Spectral Energy Distributions of T Tauri Stars with Passive Circumstellar Disks," ApJ 490, 368-+ (Nov. 1997).

[22] Masset, F., "FARGO: A fast eulerian transport algorithm for differentially rotating disks," A\&AS 141, 165-173 (Jan. 2000).

[23] Mawet, D., Riaud, P., Hanot, C., Vandormael, D., Loicq, J., Baudrand, J., Surdej, J., and Habraken, S., "The annular groove phase mask coronagraph: an achromatic optical vortex," in [SPIE , Proc. SPIE 6693, id. \#66931M (2007)], Presented at the Society of Photo-Optical Instrumentation Engineers (SPIE) Conference 6693, 52-+ (Sept. 2007). 